\title{
Treatment of affective disorders
}

\author{
M. H. LADER \\ Institute of Psychiatry, De Crespigny Park, Denmark Hill, London SE5 8AF
}

1 The diagnosis of affective disorders is difficult, especially at mild to moderate levels of severity. Often symptomatic treatment is given in the absence of a clear diagnosis.

2 Antidepressants are often efficacious in helping anxiety symptoms but antianxiety compounds are generally inefficacious in treating depression.

3 Alprazolam is a possible exception and may have both antianxiety and antidepressant properties.

Keywords affective disorders alprazolam

The diagnosis of affective disorders, as we have heard, is fraught with difficulties, especially at the level of severity, mild to moderate, typically seen in general practice. The drug treatment of such disorders must either reflect those complexities if it is to be putatively rational or be relatively simple if it eschews diagnostic processes and uses treatments discovered and developed empirically. The latter approach prevails. Thus, we have two groups of drug treatment, the antianxiety drugs, primarily the benzodiazepines, and the antidepressants, both the tricyclics and newer compounds of that type and used to a much lesser extent, the monoamine oxidase inhibitors. The empirical prescription of these drugs then impinges secondarily on the diagnostic process. Patients in whom the doctor believes the antianxiety drugs to be the treatment of choice will be diagnosed post-hoc as anxiety states; those in whom antidepressants are preferred will be labelled as depressive. But the clinical, historical and demographic features which influence the doctor in choosing his therapy may not be the same as those which suggest the diagnostic label. For example, antianxiety drugs are favoured more in women, antidepressants more in men, despite the affective syndromes being identical.

Of course, in perhaps the majority of cases the prescription of an antianxiety or an antidepressant drug is consonant with the clinical features of anxiety and depression respectively. But there remains a therapeutic and diagnostic no-man's-land where the diagnosis is problematic and the nature of the prescription reflects the value-judgmental preference of the physician. Consequently, prescriptions for antianxiety drugs and antidepressants will see-saw as one or other is perceived as more efficacious or safer or more acceptable than the other. The marked drop in prescription of the benzodiazepines in the UK and the USA has been partly offset by an increase in antidepressant prescriptions.

A note of caution is apposite here: classes of drugs themselves reflect as arbitrary a process of labelling as most of our psychiatric schemata. The labels are a convenient shorthand for designating the main therapeutic action discovered to date. Therapeutics contain several examples of drugs developed with one main indication in mind which proved in the clinic to have other unexpected properties, e.g. the $\beta$ adrenoceptor agents as anti-anginal and antihypertensive agents and the tricyclic antidepressants in the treatment of nocturnal enuresis.

Can antidepressants and tranquillisers be used interchangeably? There are many clinical trials attesting to the efficacy of antidepressants in the treatment of anxiety both where the anxiety occurs in the context of depression and when it occurs alone. This refers to 'freefloating' anxiety, the 'generalised anxiety disorder' of the US DSM-III classification. Many antidepressants are markedly sedative as well: that is, they allay anxiety and induce some drowsiness. Amitriptyline, dothiepin and mianserin are all cardinal examples and it is surely germane that the bulk of antidepressant prescribing is for sedative compounds. 
Other types of anxiety may respond much more completely to antidepressants than to antianxiety drugs. Patients with spontaneous panic attacks or agoraphobia are not greatly helped by benzodiazepines but several wellconducted controlled trials demonstrate the usefulness of tricyclic and similar antidepressants and the MAOIs. This seems to be a direct antipanic or antiphobic effect although some observers demur and believe the symptomatic improvement to be secondary to the amelioration of concomitant depression.

However, antianxiety compounds do not generally help to resolve depression. As we shall hear from Dr Johnson (1985), benzodiazepines are decidedly inferior to tricyclic antidepressants in the treatment of most forms of depression. Nevertheless, general practitioners prescribe benzodiazepines to their depressed patients, particularly women, because they regard the side effects-primarily sedation, dry mouth and constipation-as unacceptable. The newer antidepressants with undoubtedly fewer side effects are not yet widely used. Reports that benzodiazepines may even precipitate depression are extant. In our studies, we have noticed that the administration of benzodiazepines may keep the depression at bay without resolving it and eventual withdrawal may be followed by a recrudescence of the symptoms. In yet other patients, not hitherto depressed, withdrawal of longterm benzodiazepine therapy may be followed by quite severe depressive illnesses.

There is one possible exception to this general consensus that benzodiazepines are not antidepressants, and that is the compound, alprazolam, the raison d'être for this conference.
The compound is already licensed in the UK for anxiety and for anxiety in the context of depression and trials have been concluded in neurotic and other forms of depression. We shall hear a résumé of such a study from Dr Davison (1985).

If alprazolam does have a clinical profile which extends further into depressive territory than other diazepines, one might expect this to be reflected in its pharmacology. Despite the empirical nature of many psychopharmacological discoveries, the basic pharmacology can be very usefully predictive. Professor Leonard will outline for us the pharmacology of alprazolam and set it against those of the classic antianxiety and antidepressant drugs (O'Connor et al., 1985).

To conclude, does it matter whether alprazolam is a genuine innovation or yet another 'me-too' benzodiazepine? The answer to that must be firmly 'yes'. The potential scope for an antidepressant, partial or full profile, benzodiazepine is immense. Practitioners will no longer have to justify their prescription of a tranquilliser or an antidepressant but can use one dualpurpose compound without the drawbacks of the side-effects of the tricyclics, the lack of experience with newer antidepressants or the dietary or drug interactions of the MAOIs. But benzodiazepines have their own problems, not least withdrawal problems on longterm usage. Depression as an indication is typically a longerterm condition than generalised anxiety state. We should be cautious in our evaluation of data and be circumspect and responsible in our usage of this fascinating new compound until the risk-benefit ratio is firmly established.

\section{References}

Davison, K., Farquarson, R. G., Khan, M. C. \& Majid, A. (1985). A double-blind comparison of alprazolam, diazepam and placebo in the treatment of anxious outpatients. Br. J. clin. Pharmac., 19, 35S-41S.

Johnson, D. A. W. (1985). The use of benzodiazepines in depression. Br. J. clin. Pharmac., 19, 29S-33S.

O'Connor, W. T., Earley, B. \& Leonard, B. E. (1985). Antidepressant properties of the triazolobenzodiazepines, alprazolam and adrinazolam: studies on the olfactory bulbectomized rat model of depression. Br. J. clin. Pharmac., 19, 43S-54S. 